\title{
EL TURISMO ENOLÓGICO EN LA DO RIBEIRO, SITUACIÓN Y PERSPECTIVAS
}

\author{
Maria do Mar Pérez-Fra*, Ana Isabel García-Arias, Edelmiro López-Iglesias, Bernardo Valdês- \\ Paços, Iban Vázquez-González
}

Universidade de Santiago de Compostela, (Lugo), mariadomar.perez@usc.es

\begin{abstract}
Resumen
El turismo enológico es reconocido en la literatura académica como un recurso para el desarrollo rural: constituye una oportunidad comercial para las bodegas, en cuanto que les permite establecer un canal de comercialización directo y les otorga mayor notoriedad de marca, y puede ser además una oportunidad para la generación de riqueza a través de sus efectos multiplicadores sobre la economía local. La presente investigación analiza el caso una de las áreas con mayor tradición vitivinícola de España: el territorio comprendido en la DO Ribeiro (Ourense). Un espacio con una fuerte identidad histórica y territorial ligada al vino, y en el que este producto se ha convertido en un elemento vertebrador de su imagen turística. A partir del análisis de la oferta y demanda turística existente, de la realización de entrevistas a empresas y del estudio de las iniciativas que diferentes entidades han puesto en marcha para la creación de una marca enoturística reconocida, se concluye que la consolidación de la comarca de O Ribeiro como un producto enoturístico exitoso es aún una tarea pendiente y se elabora una DAFO que servirá como punto de partida para el diseño de una estrategia de mejora.
\end{abstract}

Enoturismo, Ribeiro, Vino, Desarrollo Rural, Turismo rural.

\section{Introducción y objetivos}

El enoturismo puede realizar una contribución importante a la industria del turismo y al desarrollo de nuevos paradigmas experienciales (Santos et al, 2020): se trata de una actividad que tiene un gran potencial de creación de riqueza y que permite avanzar en el desarrollo de las áreas rurales desde una perspectiva integradora y sostenible ambientalmente (Montella, 2017). Es una actividad que no solo genera ingresos adicionales para el sector vitivinícola, sino que al hacer del vino un foco de atracción turística crea sinergias entre bodegas y empresas de hostelería, constituyendo una vía para la promoción de los productos agroalimentarios locales (Brochado et al, 2021).

El turismo ligado al vino es una actividad relativamente reciente en España, y ello a pesar de hay un importante número de regiones vitivinícolas que están apostando por él (Romero, 2021). El modelo de referencia en nuestro país es el de Rutas del Vino de España, un club de producto de ámbito estatal creado por la Asociación Española de Ciudades del Vino (ACEVIN). Esta entidad junto con la Secretaría de Estado y Turismo iniciaron el proceso de definición y diseño de la metodología precisa para la creación del producto turístico Rutas del Vino de España ${ }^{68}$ (López-Guzman et al, 2013).

Son numerosos los trabajos que apuntan tanto al potencial dinamizador de esta actividad para los territorios rurales, como a que para que tenga éxito debe aglutinar la diversidad de recursos existentes en el territorio con capacidad para constituirse en atractivo turístico (Andrade-Suarez y Caamaño-Franco, 2020, Cruz-Ruiz et al 2020, López-Guzman et al, 2013, Sheridan, L. 2009). Así pues, un proyecto de enoturismo exitoso partirá de una adecuada puesta en valor de los recursos existentes en el territorio de la Denominación de Origen (DO), construyendo sobre esta base un producto destinado tanto a visitantes interesados en el vino, como a aquellos que lleguen a la zona por otros motivos y para los que el vino sea un reclamo adicional más (ACEVIN, 2018).

El objetivo de este trabajo es analizar la situación del turismo enológico en la DO Ribeiro. Para ello se realiza un estudio de la oferta y demanda y se construye una DAFO que servirá de base para el diseño de un futuro plan estratégico.

\section{Metodología}

Como punto de partida se ha llevado a cabo una revisión exhaustiva de los trabajos disponibles sobre turismo enológico en la DO Ribeiro. Una vez concluida, se ha realizado un análisis de la oferta turística existente en la zona, de las actuaciones puestas en marcha por las instituciones públicas y privadas enfocadas a la promoción y dinamización turística y, por último, de la demanda turística. La información utilizada procede de las siguientes fuentes:

- Información estadística disponible a nivel municipal en el INE, IGE y Turismo de Galicia.

\footnotetext{
${ }^{68}$ Si bien ACEVIN nace en el año 1994 la marca Rutas del Vino no surge hasta el año 2006.
} 
- Análisis de contenido de las noticias generadas por las instituciones y empresas vinculadas con la actividad turística en esta comarca desde el año 2006 al 2019, recogidas en la prensa de ámbito autonómico y local.

- Revisión de las páginas web de instituciones vinculadas con la promoción turística de la zona y de las empresas socias de la Ruta do Viño do Ribeiro.

- Análisis del material promocional elaborado o esponsorizado por la Ruta do Viño do Ribeiro.

- Entrevistas a la técnica de la Ruta do Viño do Ribeiro, al presidente de la DO y a técnicos de las 2 Oficinas Turísticas de la comarca (realizadas en octubre-noviembre 2019)

- Dinámica grupal con 8 de las 29 empresas (bodegas y hostelería) socias de la Ruta do Viño do Ribeiro, uno de los técnicos de turismo de la zona, el presidente de la DO y la técnica de la Ruta do Viño do Riveiro (noviembre 2019).

\section{Resultados}

El territorio comprendido en la DO O Ribeiro reúne buena parte de las características definidas como necesarias para crear un producto enoturístico exitoso. Es una de las DO más antiguas de España, la comarca tiene una marcada identidad territorial basada en el vino y dispone de recursos patrimoniales y naturales relevantes que permiten la construcción de un producto turístico completo y atractivo: enclaves naturales, posibilidad de actividades acuáticas, recursos termales y un rico patrimonio histórico, estrechamente vinculado con la producción vitivinícola. Por otra parte, se trata de un espacio bien comunicado tanto por carretera como por tren con las principales ciudades gallegas y del norte de Portugal, lo que le asegura una buena conectividad nacional e internacional por vía aérea. Además, en O Ribeiro desde hace más de una década distintas entidades públicas y privadas realizan actuaciones de promoción turística.

A pesar de todo ello, el análisis de la demanda realizado ha puesto en evidencia la debilidad que por el momento presenta el enoturismo en esta DO. En aras de la obligada brevedad, simplemente señalaremos que el impacto de esta actividad es reducido, si bien el número de visitantes que reciben las bodegas presenta una tendencia al alza. El año 2018 ha sido hasta el momento el de mayor número de visitantes recibidos ${ }^{69}$ con 13.343 personas. Cifra muy reducida si lo comparamos, por ejemplo, con la obtenida por la Ruta del Vino Rías Baixas: 116.557 visitantes en ese mismo año (ACEVIN 2019). Por otra parte, de acuerdo con lo recogido en la dinámica de grupo, incluso para las bodegas adheridas a la Ruta del Vino el impacto de la actividad turística en su cuenta de resultados es reducido en la mayor parte de los casos.

A partir de la información consultada y elaborada se ha construido una matriz DAFO en la que se recogen los elementos a considerar para al diseño de una estrategia de dinamización enoturística de la zona. Los elementos que integran los 4 bloques de la DAFO se han ordenado internamente utilizando tres ejes temáticos considerados relevantes:

1-Elementos relativos al diseño del producto y de la estrategia de promoción.

2-Características territoriales.

3-Características del sector vitivinícola.

Anteriormente se ha señalado que en la construcción de un producto enoturístico el territorio juega un papel relevante, siendo de hecho la base que sustenta su diseño. De ello se deriva que la existencia de colaboración público-privada, la voluntad de cooperación entre los agentes implicados y la existencia de una visión común compartida sobre la importancia del vino como recurso turístico, sean elementos importantes para el éxito o fracaso de la actividad. El trabajo de campo realizado ha puesto de manifiesto la carencia de una cultura de cooperación mínimamente arraigada. Así, en el territorio de la DO hay hasta 3 instituciones diferentes operando que tienen como objetivo la dinamización turística, si bien únicamente una de ellas tiene como finalidad exclusiva la promoción del enoturismo: Mancomunidade Turística Terras do Avia ${ }^{70}$, Xeodestino Ribeiro Carballiño ${ }^{71}$ y Ruta do Viño do Ribeiro ${ }^{72}$. El trabajo de campo realizado ha puesto de manifiesto la escasa colaboración que se ha venido estableciendo entre ellas, de hecho, no han realizado ninguna iniciativa conjunta hasta el año 2017.

Esa ausencia de cooperación entre los agentes del territorio se extiende a otros ámbitos considerados en el análisis. Las actuaciones de los distintos niveles de la Administración, instituciones y empresas implicadas

\footnotetext{
${ }^{69}$ Datos proporcionados por la Ruta do viño do Ribeiro.

${ }^{70}$ Creada en el 2000, a partir de la aprobación de un plan europeo de dinamización turística, tiene por socios a 4 de los municipios de la DO, centra su actividad en campañas de promoción y creación de pequeña infraestructura turística a través de fondos de cooperación local.

71 La ley 7/2011 del Turismo de Galicia define los Xeodestinos como espacios geográficos limítrofes que comparten una homogeneidad territorial basada en sus recursos turísticos naturales, patrimoniales y culturales, con capacidad para generar flujos turísticos y que, junto con su población, conforman una identidad turística diferenciada y singular. Creado en 2011 está integrado por 21 municipios, entre los que están 12 de la DO.

72 Esta entidad nace en el año 2006 y está integrada en la actualidad por 9 de los 14 ayuntamientos de la DO y 31 empresas, 18 de las cuales son bodegas.
} 
en la dinamización y promoción del turismo en $\mathrm{O}$ Ribeiro adolecen de falta de planificación y comunicación. Es más, tanto el análisis de los resultados de la dinámica de grupo como de los materiales promocionales, nos han permitido constatar que llegan al punto de manifestarse como claramente contradictorias en algunos momentos. Otro elemento a destacar es la baja calidad de los materiales empleados para la promoción del enoturismo y, especialmente, de las páginas web de las instituciones y empresas analizadas. Se trata ésta de una cuestión muy relevante dado que las páginas web son hoy en día un vehículo de promoción fundamental (Portela y Abad, 2020).

A la escasa disponibilidad de recursos financieros que tienen algunas de las pequeñas empresas que participan en la Ruta do Viño do Ribeiro, hay que añadir la de la propia entidad: lo reducido de su

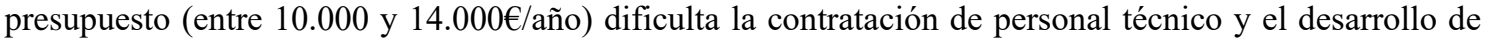
programas que vayan más allá de acciones puntuales de reducido alcance. Por otra parte, la limitación presupuestaria ha impedido que se consolidara la certificación (provocando su salida de ACEVIN en el año 2012) y dificulta la implementación no solo de acciones dirigidas a corregir debilidades, sino también de programas que aprovechen las oportunidades detectadas.

Un producto enoturístico debe contar con una entidad de gestión en la que estén representados agentes tanto de la estructura institucional como de las bodegas y empresas de hostelería del territorio (Miranda y Fernández, 2011). La función principal de esta entidad es ocuparse de la planificación, diseño y gestión del producto enoturístico. De ahí que se considere una fortaleza el hecho de que O Ribeiro cuente con una entidad constituida y con presencia en el territorio con capacidad para asumir estas funciones. Pero si bien el hecho de contar con una Ruta del Vino desde 2006 debe considerarse una fortaleza, también es necesario señalar dos debilidades que deben superarse: el número de empresas adheridas es muy reducido, y esta debilidad es especialmente evidente en cuanto a empresas que ofrecen servicios turísticos más allá de bodegas y alojamiento. A modo de ejemplo, apuntar que únicamente se cuenta con la participación de dos empresas de servicios de restauración.

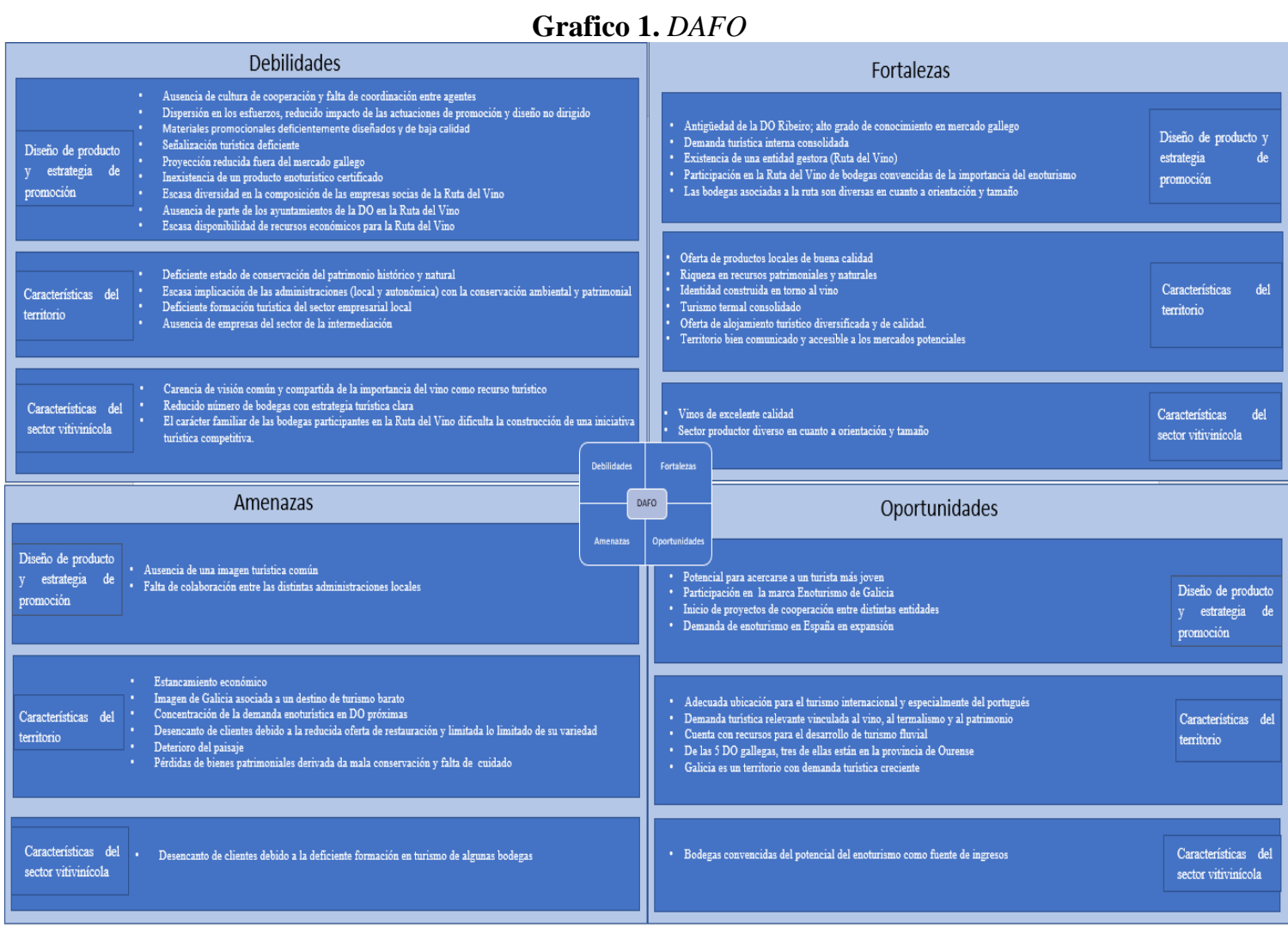

\section{Conclusiones}

El trabajo realizado nos ha permitido diseñar una serie de líneas de actuación que deberán ser priorizadas en el diseño de una estrategia para el desarrollo del enoturismo en O Ribeiro. De entre ellas son tres las que queremos destacar:

- Se ha detectado una importante debilidad financiera que dificulta la puesta en marcha de medidas de promoción y de creación de infraestructura turística efectivas. Se precisa un mayor grado de participación e implicación tanto de empresas, especialmente bodegas y restauración, como de la 
administración local del territorio. Es necesario ampliar el número de empresas participantes en la Ruta del Vino, así como conseguir una adhesión activa de aquellas que a pesar de participar como socias tienen un bajo nivel de implicación. Esto se podría facilitar con la definición de un protocolo de adhesión que regule comportamientos de las empresas socias.

- Tan importante como la escasez de recursos financieros es la falta de cooperación entre las distintas entidades públicas que operan en O Ribeiro, situación que provoca que el recurso al apoyo público no sea posible más allá de iniciativas puntuales y poco dirigidas. La ausencia de cooperación entre los agentes del territorio es en nuestra opinión uno de los principales problemas. La solución pasa forzosamente por la cooperación entre administraciones y empresas para conseguir un marco de financiamiento plurianual que permita generar un escenario en el que la planificación a medio/largo plazo sea posible. El importante número de agentes públicos y privados con intereses turísticos en el territorio es un elemento positivo, especialmente porque sus intereses declarados son coincidentes. Todo ello debería permitir consolidar las bases para el establecimiento de una estrategia coordinada que permita una mayor efectividad de los recursos.

- Para finalizar queremos resaltar que la certificación es en la actualidad el principal paraguas del enoturismo en España y por lo tanto debería ser considerada un objetivo. Se trata de una oportunidad para incrementar la difusión del Ribeiro más allá del mercado gallego, que es de donde en la actualidad procede la mayor parte de la demanda turística.

\section{Bibliografía}

ACEVIN (2019). Informe de visitantes a bodegas y museos del vino, https://wineroutesofspain.com/wpcontent/uploads/2020/10/informe-de-visitantes.pdf

ACEVIN (2018). Análisis de la Demanda Turística Rutas del Vino de España 2017-2018, https://wineroutesofspain.com/wp-content/uploads/2021/04/analisis-demanda-turistica-2018-2019.pdf Andrade-Suárez, M., \& Caamaño-Franco, I. (2020). The relationship between industrial heritage, wine tourism, and sustainability: A case of local community perspective Sustainability. 2020; 12(18):7453. https://doi.org/10.3390/su12187453.

Cruz-Ruiz E, Zamarreño-Aramendia G, Ruiz-Romero de la Cruz E. Key Elements for the Design of a Wine Route. The Case of La Axarquía in Málaga (Spain). Sustainability. 2020; 12(21):9242. https://doi.org/10.3390/su12219242.

Hall, C. M., \& Mitchell, R. (2000). Wine tourism in the Mediterranean: A tool for restructuring and development. Thunderbird International Business Review, 42(4), 445-465.

López-Guzmán, T., García, J. R., \& Rodríguez, Á V. (2013). Revisión de la literatura científica sobre enoturismo en España. Cuadernos De Turismo, (32), 171-188.

Miranda, B., \& Fernández, R. (2011). Vino, turismo e innovación: Las rutas del vino de españa, una estrategia integrada de desarrollo rural. Estudios De Economía Aplicada, 29(1), 129-165.

Montella, M. M. (2017). Wine tourism and sustainability: A review. Sustainability, 9(1), 113.

Portela, J. F., \& Abad, C. J. P. (2020). Las plataformas digitales en el desarrollo del enoturismo en Castilla y León: páginas web y redes sociales. Cuadernos de Turismo, (46), 47-69.

Romero Ligero, M. A.Modelos de enoturismo y turismo gastronómico en españa. In Compés López, R. Szolnoki G. (Ed.), Enoturismo sostenible e innovador. modelos de éxito alrededor del mundo (pp. 125139). Almeria: Caja Mar.

Santos, V., Ramos, P., Almeida, N., \& Santos-Pavón, E. (2020). Developing a wine experience scale: A new strategy to measure holistic behaviour of wine tourists. Sustainability, 12(19), 8055.

Sheridan, L., Alonso, A. D., \& Scherrer, P. (2009). Wine tourism as a development initiative in rural canary island communities. Journal of Enterprising Communities (3), 291-305. 